\title{
DOMAIN PERTURBATION FOR PARABOLIC EQUATIONS
}

\author{
PARINYA SA NGIAMSUNTHORN
}

(Received 16 September 2011)

2010 Mathematics subject classification: primary 35B20; secondary 35K10, 35K20, 35K58, 35K90, 37L05, 49J40.

Keywords and phrases: bounded entire solutions, domain perturbation, initial-boundary value problems, invariant manifolds, parabolic equations.

Domain perturbation for boundary value problems is an important topic in the analysis of partial differential equations. It has, for instance, been used in constructing examples where the solution structure of nonlinear problems is much more complicated than when the domain is a ball (see, for example, [3, 4]). An application of domain perturbation also appears in shape optimisation problems or numerical analysis. Domain perturbation has been extensively studied for elliptic equations (see the survey of Daners [8]). The aim of this work is to develop a general theory of domain perturbation for linear and semilinear parabolic equations of second order. Let $\Omega$ be a bounded open set in $\mathbb{R}^{N}, N \geq 2$. We consider a sequence of domains $\Omega_{n}$ in $\mathbb{R}^{N}$ as a perturbation of $\Omega$ and study the perturbed parabolic equation

$$
\begin{cases}\frac{\partial u}{\partial t}+\mathcal{A}_{n}(t) u=f_{n}(x, t, u) & \text { in } \Omega_{n} \times(0, T], \\ \mathcal{B}_{n}(t) u=0 & \text { on } \partial \Omega_{n} \times(0, T], \\ u(\cdot, 0)=u_{0, n} & \text { in } \Omega_{n},\end{cases}
$$

where $\mathcal{A}_{n}$ is an elliptic operator of the form

$$
\mathcal{A}_{n}(t) u:=-\partial_{i}\left(a_{i j, n}(x, t) \partial_{j} u+a_{i, n}(x, t) u\right)+b_{i, n}(x, t) \partial_{i} u+c_{0, n}(x, t) u
$$

and $\mathcal{B}_{n}$ is one of the following boundary operators:

$$
\begin{aligned}
& \mathcal{B}_{n}(t) u:=u \quad \text { Dirichlet boundary condition, } \\
& \mathcal{B}_{n}(t) u:=\left(a_{i j, n}(x, t) \partial_{j} u+a_{i, n}(x, t) u\right) v_{i} \quad \text { Neumann boundary condition. }
\end{aligned}
$$

Thesis submitted to The University of Sydney, March 2011. Degree approved, August 2011. Supervisor: Dr Daniel Daners; Associate Supervisor: Professor E. N. Dancer. The thesis can be accessed online at http://hdl.handle.net/2123/7775.

(c) 2011 Australian Mathematical Publishing Association Inc. 0004-9727/2011 \$16.00 
Here, we use the summation convention with $i, j$ running from 1 to $N$. The boundary condition is considered in a weak sense because the (outer) unit normal vector $v=\left(v_{1}, \ldots, v_{N}\right)$ may not exist. We focus on the case of singular perturbations of the domain so that it is not possible in general to apply a change of variables (coordinate transform) to change the perturbed equation into an equivalent problem over the same domain $\Omega$. Hence, this class of domain perturbations cannot be reduced to ordinary perturbation theory for the coefficients. The two main aspects considered in this work are the behaviour of solutions and the dynamical behaviour under changes of the domain.

For the behaviour of solutions, we establish the convergence of solutions when the domains converge in a certain sense. Indeed, the concept of Mosco convergence of domains is adopted (see $[2,6,9]$ ).

We first consider initial value problems for linear parabolic equations. It is known that the convergence of solutions of linear autonomous parabolic equations under domain perturbation can be obtained from the strong convergence of the resolvents of the corresponding elliptic operators and semigroup theory based on the crucial result of Arendt [1, Theorem 5.2]. We show that there is also a relation between domain perturbation for nonautonomous linear parabolic equations and domain perturbation for elliptic equations by establishing several equivalent descriptions of Mosco convergence. Using this abstract result, the work of Daners [5] for Dirichlet problems is extended to cover the more difficult case of Neumann problems, and more general classes of domain perturbations. This gives rather general results on domain perturbation for linear nonautonomous equations. A similar technique can be applied to obtain the convergence of weak solutions of parabolic variational inequalities when the underlying convex set is perturbed. These results are included in [11].

Based on the linear theory developed above and the work of Daners [7], we then consider initial value problems for semilinear parabolic equations. We prove the convergence of solutions of Dirichlet or Neumann problems under uniform estimates for the evolution systems of the linear problems and a uniform Lipschitz condition for the nonlinearities. The result also contains an improvement of [7, Theorem 4.6], which requires a weaker assumption of pointwise convergence for the semilinear terms instead of a uniform convergence.

Besides the convergence of solutions of initial value problems, we are also interested in the behaviour of bounded entire solutions (solutions that are defined for all time $t \in(-\infty, \infty)$ and are bounded in a suitable function space) under domain perturbation. Assuming the existence of an exponential dichotomy, bounded entire solutions for linear parabolic equations can be represented in terms of a heat kernel. By using the representation formula and the results for initial value problems, we obtain the convergence of bounded entire solutions for autonomous linear problems subject to a Dirichlet boundary condition in the $L^{2}$-setting. The convergence result is then extended to the $L^{p}$-framework by interpolation arguments. For the $L^{p}$-theory, we also prove Hölder regularity of bounded entire solutions with respect to time. Although a similar regularity of solutions is available for evolution equations in fractional power 
spaces or interpolation spaces, the same argument cannot be applied when we work in the $L^{p}$ scales. In addition, we obtain some results on the persistence of a class of bounded entire solutions for semilinear parabolic equations using topological degree theory. The new result for this part has been published in [10].

The second aspect concerns the dynamical behaviour of parabolic equations under domain perturbation. In this part, we consider semiflows induced by solutions of semilinear parabolic equations subject to a Dirichlet boundary condition in the $L^{2}$ framework and study the persistence of invariant manifolds near an equilibrium solution. It is well known from the theory of dynamical systems that hyperbolicity of an equilibrium is the main concept for persistence under small perturbations. We show that this principle is also valid for singular domain perturbation. Indeed, we obtain the upper and lower semicontinuity of the local stable invariant manifolds and the local unstable invariant manifolds near a hyperbolic equilibrium of the limit equation under Mosco convergence of the domains (see [12]).

\section{References}

[1] W. Arendt, 'Approximation of degenerate semigroups', Taiwanese J. Math. 5(2) (2001), 279-295.

[2] D. Bucur and N. Varchon, 'Boundary variation for a Neumann problem', Ann. Sc. Norm. Super. Pisa Cl. Sci. 29(4) (2000), 807-821.

[3] E. N. Dancer, 'The effect of domain shape on the number of positive solutions of certain nonlinear equations', J. Differential Equations 74(1) (1988), 120-156.

[4] E. N. Dancer, 'The effect of domain shape on the number of positive solutions of certain nonlinear equations. II', J. Differential Equations 87(2) (1990), 316-339.

[5] D. Daners, 'Domain perturbation for linear and nonlinear parabolic equations', J. Differential Equations 129(2) (1996), 358-402.

[6] D. Daners, 'Dirichlet problems on varying domains', J. Differential Equations 188(2) (2003), 591-624.

[7] D. Daners, 'Perturbation of semi-linear evolution equations under weak assumptions at initial time', J. Differential Equations 210(2) (2005), 352-382.

[8] D. Daners, 'Domain perturbation for linear and semi-linear boundary value problems', in: Handbook of Differential Equations: Stationary Partial Differential Equations, Vol. 6 (ed. M. Chipot) (Elsevier, North Holland, Amsterdam, 2008), pp. 1-81.

[9] U. Mosco, 'Convergence of convex sets and of solutions of variational inequalities', Adv. Math. 3 (1969), 510-585.

[10] P. Sa Ngiamsunthorn, 'Persistence of bounded solutions of parabolic equations under domain perturbation', J. Evol. Equ., in press, doi 10.1007/s00028-011-0121-3.

[11] P. Sa Ngiamsunthorn, 'An abstract approach to domain perturbation for parabolic equations and parabolic variational inequalities', Preprint, arXiv:1109.3257.

[12] P. Sa Ngiamsunthorn, 'Invariant manifolds for parabolic equations under perturbation of the domain', Preprint, arXiv:1109.3260.

PARINYA SA NGIAMSUNTHORN, School of Mathematics and Statistics, University of Sydney, NSW, 2006, Australia

e-mail: pasa4391@uni.sydney.edu.au 